Review Article

\title{
Evaluation of Self-Directed Learning in Nursing Students: A Systematic Review and Meta-Analysis
}

\author{
Mohammad Nazarianpirdosti, ${ }^{1}$ Maryam Janatolmakan $\mathbb{D}^{2},{ }^{2}$ Bahareh Andayeshgar $\mathbb{D}^{\mathrm{D}},{ }^{3}$ \\ and Alireza Khatony $\mathbb{D}^{2,4}$ \\ ${ }^{1}$ Student Research Committee, Kermanshah University of Medical Sciences, Kermanshah, Iran \\ ${ }^{2}$ Social Development and Health Promotion Research Center, Health Institute, Kermanshah University of Medical Sciences, \\ Kermanshah, Iran \\ ${ }^{3}$ School of Health, Kermanshah University of Medical Sciences, Kermanshah, Iran \\ ${ }^{4}$ Infectious Diseases Research Center, Imam Reza Hospital, Kermanshah University of Medical Sciences, Kermanshah, Iran \\ Correspondence should be addressed to Alireza Khatony; akhatony@gmail.com
}

Received 1 October 2021; Revised 6 November 2021; Accepted 9 November 2021; Published 20 November 2021

Academic Editor: Ehsan Namaziandost

Copyright (C 2021 Mohammad Nazarianpirdosti et al. This is an open access article distributed under the Creative Commons Attribution License, which permits unrestricted use, distribution, and reproduction in any medium, provided the original work is properly cited.

\begin{abstract}
Background. Self-directed learning plays an important role in nursing education and is associated with academic achievement, communication self-efficacy, assertiveness, responsibility, and students' clinical competencies. This study was conducted to analyze the existing research on the level of self-directed learning in nursing students. Methods. In this systematic review and meta-analysis, all studies that had examined the level of self-directed learning in nursing students until March 2, 2020, were searched in Science Direct, Ovid, Scopus, PubMed, ProQuest, Web of Science, and Google Scholar databases. In the meta-analysis section, $I^{2}$ index and the random effects model were used. Comprehensive Meta-Analysis software (version 2) was used for data analysis. Results. The mean score of self-directed learning in 12 articles with 3830 samples was $156.73 \pm 1.47$ out of 200 (95\% CI: 153.3-160.1). These scores were $153.50 \pm 2.71$ (95\% CI: 148.18-158.82) and $154.67 \pm 1.32$ (95\% CI: 157.50-159.32) in the male and female students, respectively. There was a significant relationship between self-directed learning and sample size, year of study, and semester $(p<0.05)$. Conclusion. The mean self-directed learning in nursing students was at a moderate level, which does not seem to be sufficient. Given the positive role of self-directed learning in nursing education, it is essential that nursing professors teach self-directed learning skills to students via training courses.
\end{abstract}

\section{Introduction}

One of the important aspects of learning is self-directed learning (SDL) [1]. Self-directed learning is a process in which a person is active in the learning process in terms of metacognitive, motivational, and behavioral dimensions [2]. Knowles has defined SDL as "the process by which individuals take the initiative, with or without the help of others, in identifying their learning needs, setting learning goals, identifying human and material resources for learning, selecting and implementing appropriate learning strategies, and evaluating the learning outcomes" $[3,4]$.
Self-directed learning has also been suggested as an important influential factor in learning in different age groups [5]. Self-directed learners control their learning experiences using a variety of cognitive or metacognitive strategies that lead to active participation in the learning process [6]. These learners are self-motivated, diligent, independent, self-disciplined, self-confident, and goal-oriented [7]. With the growing trend of continuous and rapid changes in medical sciences and the need to prepare nursing students for lifelong learning, SDL theory has been increasingly used as a necessity in the context of nursing education [8]. Today, nursing programs focus on the use of various adult learning methods, including SDL [9]. Since 
nurses are constantly faced with the challenges of ongoing social and scientific changes in the health care domain [10], it is necessary to evaluate SDL in this field all over the world for reasons such as increasing evidence, complexity of patients' problems, and limited training time [11]. Students who are self-directed in learning also take responsibility for their own learning needs and goals [12], and this feature helps them achieve professional competencies in nursing [13]. On the other hand, SDL can be used as an indicator to predict students' academic success [12].

Evidence suggests that participation in lifelong learning through the SDL approach leads to successful adaptation to the healthcare system [14]. In the field of nursing, knowledge changes dynamically, so nursing education is trying to prepare nursing students as future nurses for lifelong learning [15]. SDL strategy in nursing students promotes their knowledge and management skills, encourages lifelong learning, and also enables them to provide safe nursing care [16]. Regarding the level of SDL in nursing students, several studies have been conducted in different countries with conflicting results. In this regard, the level of SDL in nursing students was reported to be high in Thailand, Saudi Arabia, and Turkey [17-19], but in a study in Australia, this rate was low [20]. The results of a study conducted in Iran showed that the rate of SDL was moderate [21].

Considering the importance of SDL in nursing education and the need to discover the extent of this type of learning in nursing students as future caregivers, this systematic review and meta-analysis was conducted to analyze the existing research on the level of SDL in nursing students.

This study sought to answer the following questions. (1) What is the level of SDL in nursing students? (2) What is the level of SDL by gender, semester, country, and year of study?

1.1. Theoretical Framework. There is no specific theoretical perspective to explain the concept of SDL [22]. However, Arsic refers to two theories regarding SDL: Piaget's cognitive development and Vygotsky's sociocultural theory [23]. According to Piaget's theory of cognitive development, humans are active, curious, and inquisitive throughout their lives, and curiosity is the key to gaining knowledge and meaningful learning of new things. Piaget believes that advanced forms of cognition are constructed through the activity of learners [24]. According to Vygotsky's theory, man is an active and constructive being and actively participates in teaching and learning. In this theory, the teacher also plays a role in facilitating the learners' learning process by giving feedback, using rewards, and asking and answering questions [25].

\section{Materials and Methods}

In this systematic review and meta-analysis, all studies on SDL published until March 2, 2020, were searched in the Science Direct, Ovid, Scopus, PubMed, ProQuest, Web of Science, and Google Scholar databases. The key terms used for searching included self-directed learning, self-regulated learning, lifelong learning, nursing, and students. All combinations of these key terms were also searched. No restrictions were placed on the search process, and all articles were transferred to the EndNote 8 software (for Windows, Thomson Reuters). In order to maximize the comprehensiveness of the search, the bibliography of all the obtained articles was also manually reviewed. The search strategy was as follows ("Self-directed learning" [tiab] OR "self-regulated learning"[tiab] OR "Lifelong learning”[tiab]) AND ("nursing”[tiab]) AND (“students"[tiab]).

2.1. Inclusion Criteria. All observational studies, studies that referred to the level of self-directed learning, as well as studies whose full text was available were included in the study.

2.2. Exclusion Criteria. Case studies, duplicate studies, studies with insufficient data, unrelated studies, and studies whose methodology was unclear were excluded.

2.3. Selection Process. After the search was completed, a list of abstracts of all articles was prepared, and the specifications of the articles, including the names of the journals and authors, were hidden. In this stage, which was the screening phase, the abstracts of all articles were given to two reviewers (the first and last authors). The reviewers reviewed the abstracts independently. At this point, a number of articles were excluded based on the inclusion and exclusion criteria. In the next stage, which was the eligibility evaluation, the full texts of the articles selected in the screening stage were provided to the reviewers to evaluate independently, following which the irrelevant articles were excluded. If an article was rejected, the reason was stated. In case of disagreement between the two reviewers, the article was reviewed and confirmed by the third reviewer.

2.4. Quality Assessment. The Strengthening the Reporting of Observational Studies in Epidemiology (STROBE) checklist was used to review the studies. This checklist consists of 22 items, 18 of which are general and used for a variety of observational studies, including cohort, case-control, and cross-sectional studies. There are also 4 specific sections that depend on the type of study and different aspects of methodology [26]. The maximum score in this checklist is 32. In this study, articles with a score equal to or higher than 16 were regarded as articles of average and good quality and were included in the study. Articles with scores less than 16 were excluded from the study.

2.5. Data Extraction. The data of all final articles were fed into a standard checklist. The checklist items included the name of the first author, type of study, year of publication, place of study, sample size, age, gender, level of self-directed learning, and semester, which were extracted by two judges independently. 


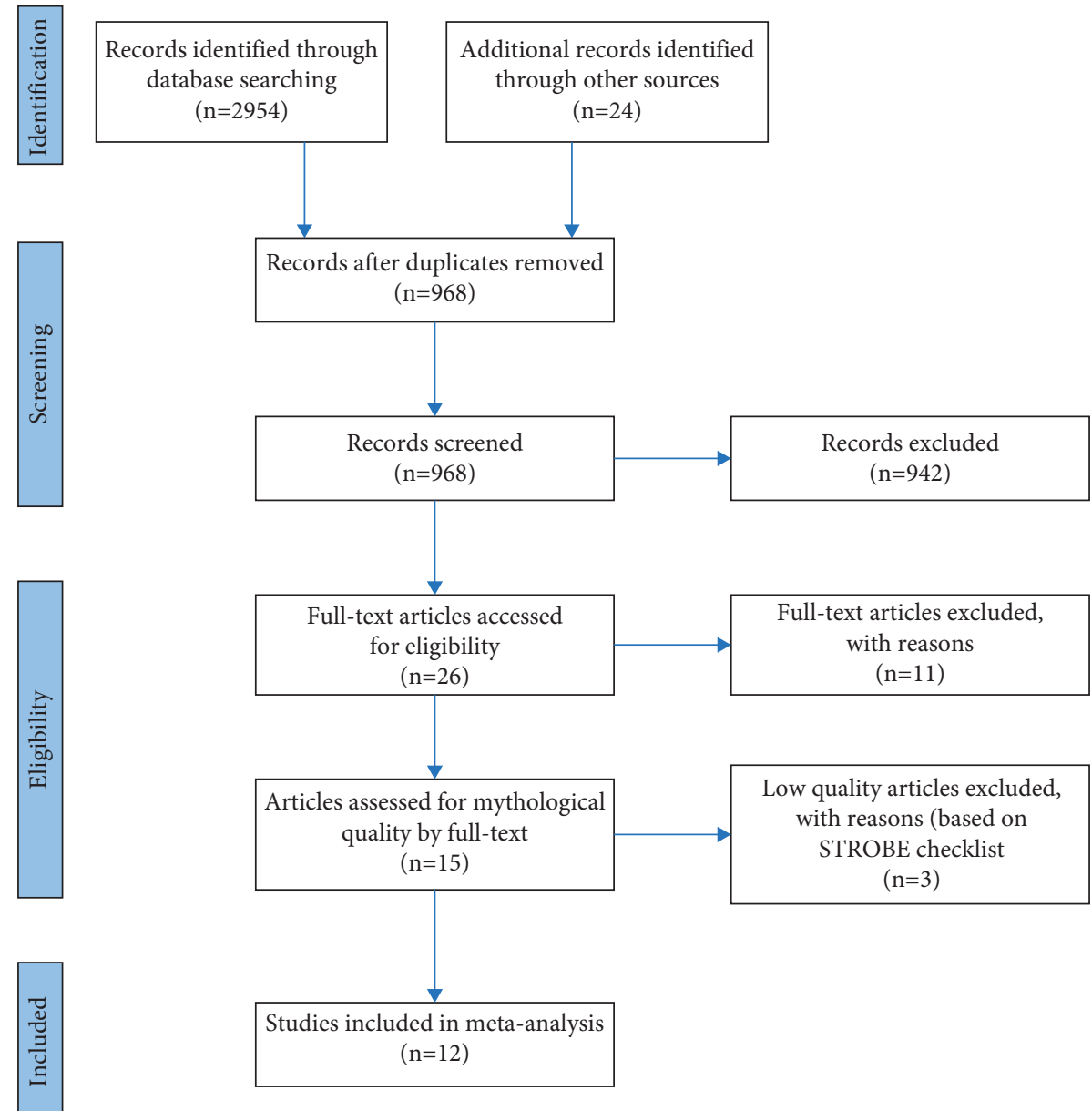

FIgURE 1: The flowchart of stages of inclusion of studies in the systematic review and meta-analysis (PRISMA 2009).

2.6. Statistical Analysis. Heterogeneity assessment of studies was performed with $I^{2}$ tests. $I^{2}$ less than $30 \%$ was regarded as low heterogeneity, $30-70 \%$ as moderate, and more than $70 \%$ as severe. Due to heterogeneity in the studies, the random effects model was used to combine them. Begg and Mazumdar test with the significance level of 0.1 was performed to examine the publication bias. Sensitivity analysis was conducted to ensure the consistency of the results. To investigate the effects of potential factors such as sample size, year of study, and semester on the heterogeneity of studies, meta-regression was used. Comprehensive Meta-Analysis software (version 2) was used to analyze the studies. Significance level was considered less than 0.05 .

\section{Results}

In accordance with the PRISMA guidelines, the extracted data were evaluated through a systematic review and meta-analysis. The initial search of the databases yielded 2954 related articles, which were then transferred to the EndNote Bibliography Management Software. Furthermore, 24 articles from the references of other studies were added to these articles. Of the 2,978 articles identified, 2010 were duplicates, which were removed. Out of the remaining 968 articles, 848 articles were excluded after initial evaluation based on the inclusion and exclusion criteria. Then, 120 articles were included in the critical evaluation stage, and finally, 105 articles were removed for reasons such as irrelevance, lack of information required, or lack of access to full text. In the final stage, according to the STROBE checklist, three articles were excluded due to low quality. Finally, twelve articles were analyzed (Figure 1).

Due to the heterogeneity of studies based on $I^{2}$ test, the random effects model was used to determine the mean score of self-directed learning. This heterogeneity might be related to year of study, place of study, measurement error, sample size, or semester. The total sample size was 3830 participants. The largest sample size was allocated to the study of Yang and Jiang with 519 participants [10]. Moreover, all studies were of average quality (Table 1). According to the Begg and Mazumdar test (significance level of 0.01 ), the publication bias was not significant $(p=0.172)$. The results of the present study showed that the mean score of self-directed learning in nursing students was $156.73 \pm 1.47$ out of 200 (95\% CI: 153.3-160.1) (Figure 2). The midpoint of each piece was equal to the average self-directed learning score in each study, and the ellipse represents the average of all studies. 


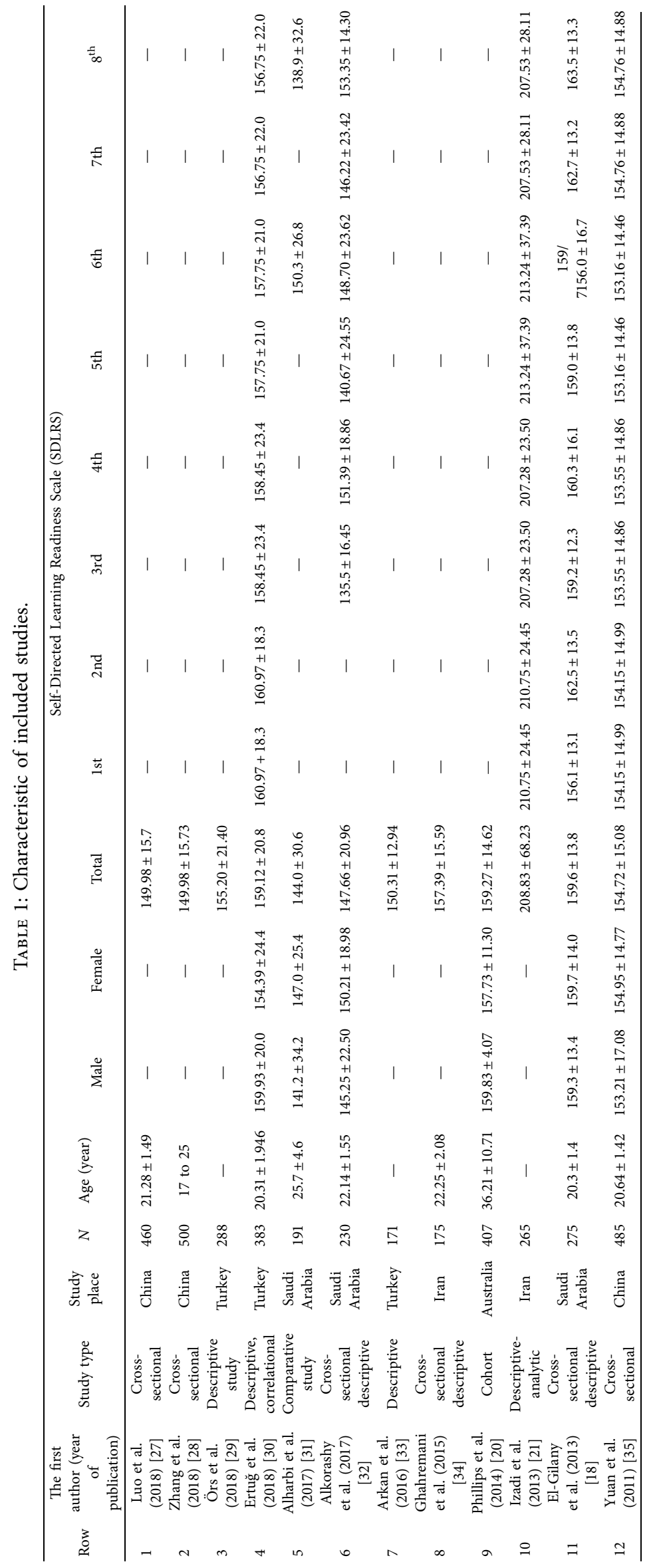




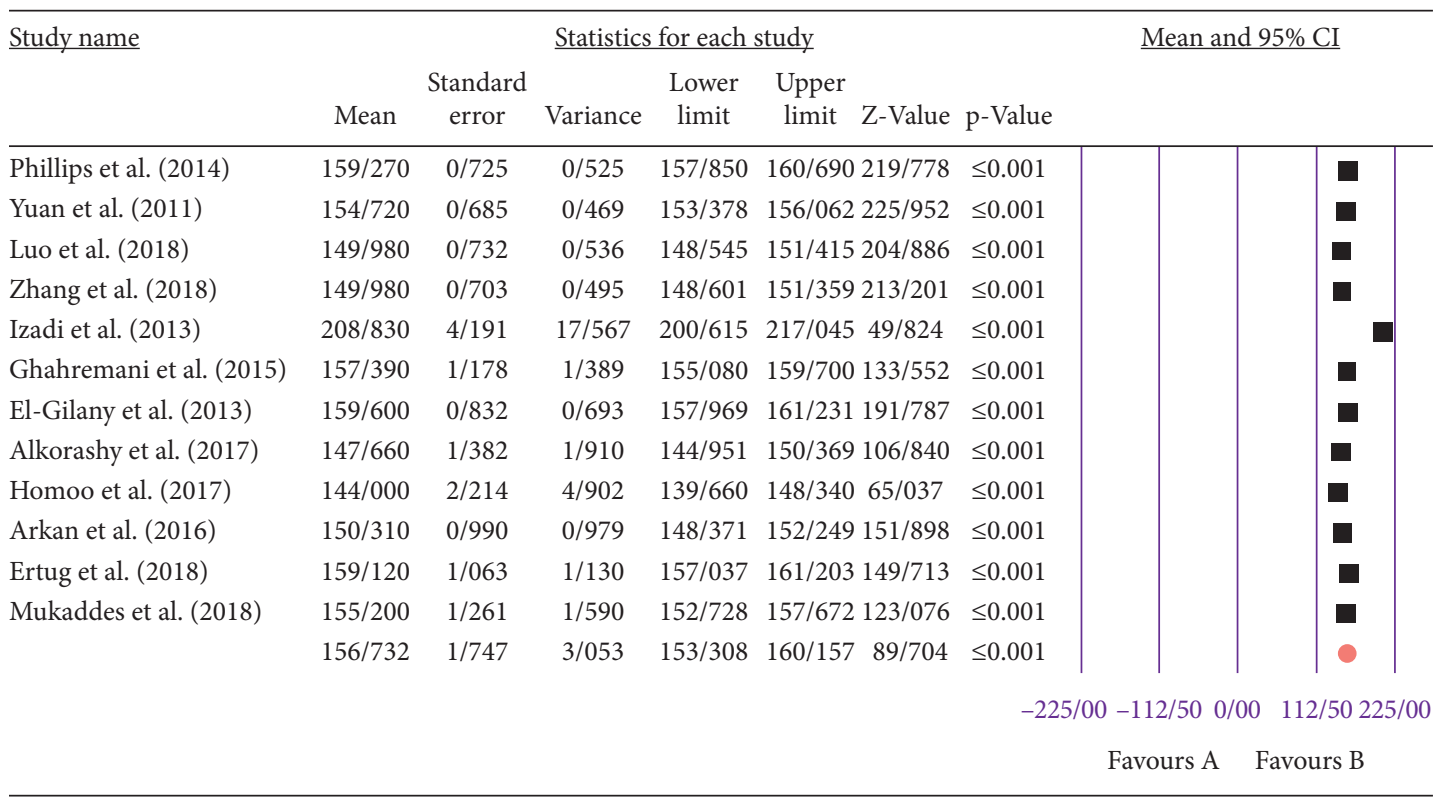

FIgURE 2: The overall mean score of self-directed learning in nursing students.

3.1. Subgroup Analysis. The overall mean scores of self-directed learning in the female and male students were $154.67 \pm 1.32$ (95\% Cl: $157.50-159.32)$ and $153.50 \pm 2.71$ (95\% Cl: 148.18-158.82), respectively, which was higher in the female students. In terms of semester, the students of the second and eighth semesters, with the mean scores of $171.98 \pm 9.89$ (95\% Cl: $152.50-191.30)$ and $162.19 \pm 6.40$ (95\% Cl: 149.60-174.70), had the highest and lowest mean scores of SDL. In terms of the country of study, the highest and lowest mean scores of learning self-directed were related to the Iranian and Saudi students, respectively, with the mean scores of $151.56 \pm 1.60$ and $150.54 \pm 7.64$, respectively (Table 2).

3.2. Metaregression. The results showed that the mean SDL decreased significantly with an increase in the sample size $(p=0.004)$. As the semester increased, the mean SDL decreased significantly $(p<0.001)$. Furthermore, the mean score of SDL increased significantly with an increase in the year of study $(p<0.001)$.

3.3. Sensitivity Analysis. Sensitivity analysis was used to ensure the consistency of the results. No changes in the results were seen after omission of each study (Figure 3).

\section{Discussion}

According to the results of the present study, the mean score of SDL was moderate in the nursing students. SDL skills are essential for the success of learners in the nursing profession, and students should learn these skills for deeper and more lasting learning [36]. Using the SDL process, the nursing students can review and improve their learning process [37]. Evidence suggests that SDL has a positive role in nursing education and is significantly associated with academic achievement [19], professional competence and communication self-efficacy [38], assertiveness and accountability [39], and clinical competencies [40]. Given the importance of SDL in nursing education, nursing educators should make an effort to persuade students to use SDL skills effectively.

Moreover, the results of the present study indicated that the mean score of SDL was higher in the female than male students. The results of some studies have indicated that female students use learning strategies more than male students [41, 42]. However, the results of a study by Lee et al. showed that the rate of SDL was higher in the male students [37]. Evidence suggests that women have a higher learning ability than men [43-45]. The reason for this difference in learning rate can be attributed to factors such as learning strategies, time management, learning motivation, and planning [46]. It seems that using psychological interventions can be helpful to increase the male students' desire for self-directed learning.

Another result of the present study was students' reduced SDL with an increase in the semester. The level of SDL readiness is expected to increase with a rise in semester because students in higher semesters have more clinical experience and decision-making power and can discuss and share their opinions in specific clinical situations [29]. The decrease in the mean SDL with a rise in the academic semester can be due to the fact that students in the first academic years have a high level of SDL due to their high motivation for learning. However, as the number of semesters increases, the desire for SDL may decrease due to the nature of the learning process in the minds of the learners.

The results of the present study indicated that the mean score of SDL varies in different countries. In this regard, the results of a study in Iran showed that the average scores of readiness for SDL in nursing students were moderate [21]. Studies in Thailand, Saudi Arabia, and Turkey have shown a high degree of readiness for SDL [17-19]. The results of a 
TABLE 2: Subgroup analysis on the country of study and mean of self-directed learning in nursing students.

\begin{tabular}{lccccc}
\hline Country & Number of articles & Sample size & $I^{2}$ & Begg and Mazumdar & Mean \pm SD $(95 \%$ CI $)$ \\
\hline Australia & 1 & 407 & 0.0 & - & $159.27 \pm 0.72(157.8-219.7)$ \\
China & 3 & 1445 & 95.53 & 0.333 & $151.56 \pm 1.60(148.4-154.7)$ \\
Iran & 2 & 440 & 99.28 & - & $182.95 \pm 25.72(132.5-233.3)$ \\
Saudi Arabia & 3 & 695 & 97.59 & 0.333 & $150.54 \pm 7.64(149.5-160.2)$ \\
Turkey & 3 & 842 & 94.61 & 0.333 & $154.86 \pm 2.73(149.5-160.5)$ \\
\hline
\end{tabular}

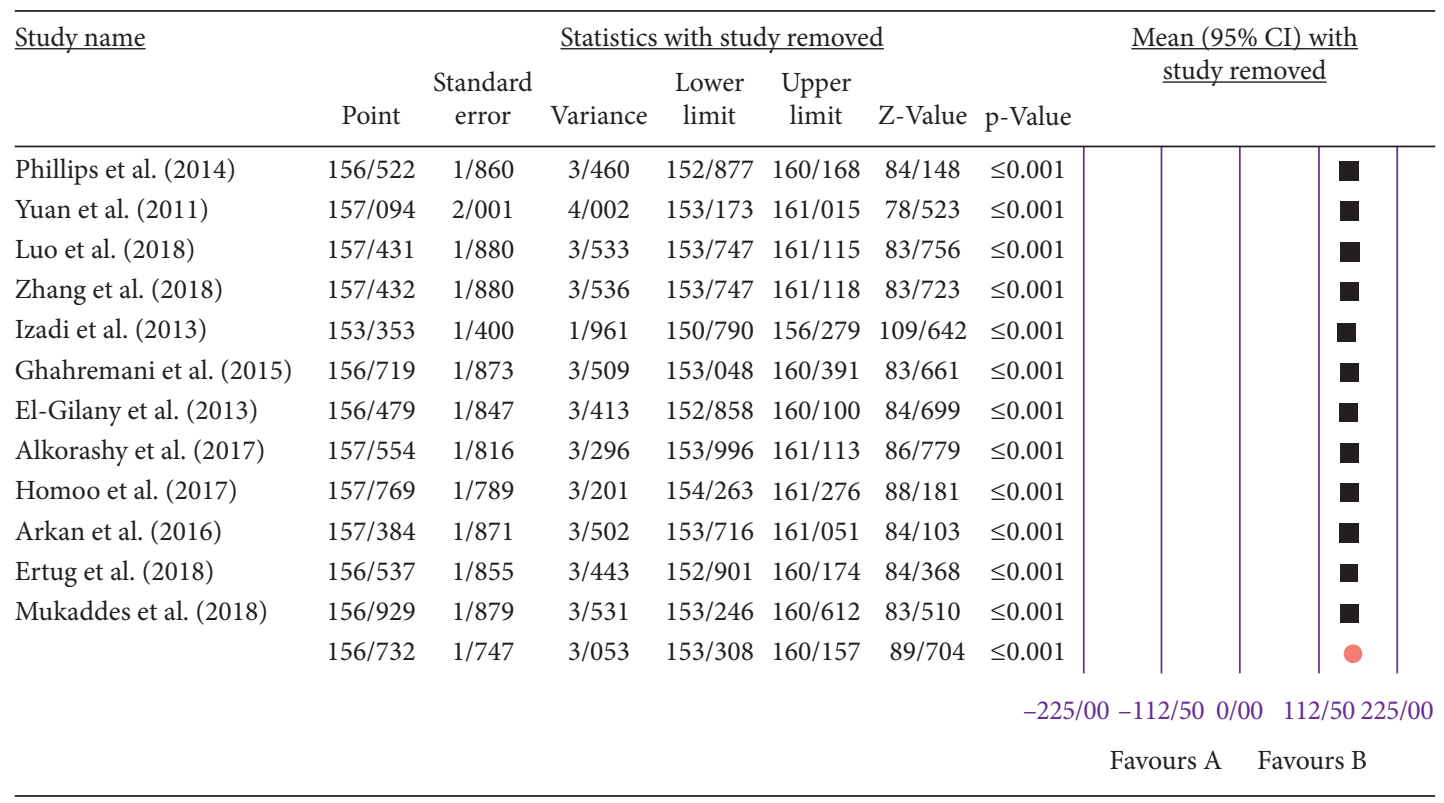

FIGURE 3: Results of sensitivity analysis.

study in Australia showed a low level of SDL in the first-year nursing students [20]. The reason for this difference can be due to the various learning cultures in different countries. Culture plays an important role in learning and cannot be ignored [47]. Learning styles are often based on culture, and students in different cultures have different patterns of learning, thinking, and behavior that affect their learning $[48,49]$. In addition, educational systems are specific and unique to each country [50]. The educational system policies can have a significant impact on students' learning in different countries [51].

4.1. Limitations. There are several tools available to measure self-directed learning, which differ in how they are scored. However, the number of these studies, after secondary evaluation, was not sufficient to perform a separate metaanalysis on them. Therefore, in the present study, only studies that used the SDL Readiness Scale were selected.

\section{Conclusion}

In order to enhance professional competence in nursing students, self-directed learning (SDL), which is of special importance, should be considered by professors and educational administrators in nursing schools. The results of this study showed that the SDL in nursing students was at a moderate level. Since SDL is one of the main pillars of problem-solving ability and plays a major role in building clinical competence in nursing students, necessary measures should be taken to promote this type of learning. In this regard, the nursing professors need to teach SDL skills to students through training courses before including them in the curriculum. The results of this study can be used to improve the quality of education and improve the teaching methods of nursing professors through the use of problembased and student-centered curriculum. Since the level of SDL in nursing students is far from the desired level, future studies are suggested to evaluate the effect of educational measures on its rate. Qualitative studies are also required to investigate factors facilitating and inhibiting SDL.

\section{Data Availability}

The identified datasets analyzed during the current study are available from the corresponding author on a reasonable request.

\section{Ethical Approval}

The ethics committee of Kermanshah University of Medical Sciences approved the study with the code IR.KUMS.REC.1398.736.

\section{Consent}

Not applicable. 


\section{Conflicts of Interest}

The authors declare there are no conflicts of interest.

\section{Authors' Contributions}

MNP, MJ, BA, and AK contributed to designing of the study. MNP and MJ collected the data, and BA analyzed the data. MNP, MJ, BA, and AK wrote the final report and manuscript. All the authors read and approved the version for submission.

\section{Acknowledgments}

This study was conducted with the financial support of Kermanshah University of Medical Sciences (no. 980642).

\section{References}

[1] A. Kaur, P. Lakra, and R. Kumar, "Self-directed learning readiness and learning styles among nursing undergraduates," Nursing and Midwifery Research Journal, vol. 16, no. 1, pp. $40-50,2020$.

[2] K. K. Cho, B. Marjadi, V. Langendyk, and W. Hu, "The selfregulated learning of medical students in the clinical environment-a scoping review," BMC Medical Education, vol. 17 , no. 1, p. 112, 2017.

[3] M. Soliman and G. Al-Shaikh, "Readiness for self-directed learning among first year Saudi medical students: a descriptive study," Pakistan Journal of Medical Sciences, vol. 31, no. 4, pp. 799-802, 2015.

[4] C. E. Slater and A. Cusick, "Factors related to self-directed learning readiness of students in health professional programs: a scoping review," Nurse Education Today, vol. 52, pp. 28-33, 2017.

[5] Y. Zhu, W. Au, and G. Yates, "University students' selfcontrol and self-regulated learning in a blended course," The Internet and Higher Education, vol. 30, pp. 54-62, 2016.

[6] M. K. Robb, "Self-regulated learning: examining the baccalaureate millennial nursing student's approach," Nursing Education Perspectives, vol. 37, no. 3, pp. 162-164, 2016.

[7] H.-J. Lai and C.-Y. Wang, "Examining public librarians' information literacy, self-directed learning readiness, and e-learning attitudes: a study from Taiwan," Malaysian Journal of Library \& Information Science, vol. 17, no. 2, pp. 101-115, 2017.

[8] S.-F. Cheng, C.-L. Kuo, K.-C. Lin, and J. Lee-Hsieh, "Development and preliminary testing of a self-rating instrument to measure self-directed learning ability of nursing students," International Journal of Nursing Studies, vol. 47, no. 9, pp. 1152-1158, 2010.

[9] E. O'Shea, "Self-directed learning in nurse education: a review of the literature," Journal of Advanced Nursing, vol. 43, no. 1, pp. 62-70, 2003.

[10] G.-F. Yang and X.-Y. Jiang, "Self-directed learning readiness and nursing competency among undergraduate nursing students in Fujian province of China," International Journal of Nursing Sciences, vol. 1, no. 3, pp. 255-259, 2014.

[11] L. Cadorin, V. Bressan, and A. Palese, "Instruments evaluating the self-directed learning abilities among nursing students and nurses: a systematic review of psychometric properties," BMC Medical Education, vol. 17, no. 1, p. 229, 2017.
[12] A. Yousefy and M. Gordanshekan, "A review on development of self-directed learning," Iranian Journal of Medical Education, vol. 10, no. 5, pp. 776-783, 2011.

[13] J.-J. Yang and M.-Y. Park, "The relationship of clinical competency and self-directed learning in nursing students," The Journal of Korean Academic Society of Nursing Education, vol. 10, no. 2, pp. 271-277, 2004.

[14] K. Chakkaravarthy, N. Ibrahim, M. Mahmud, and M. R. Venkatasalu, "Predictors for nurses and midwives' readiness towards self-directed learning: an integrated review," Nurse Education Today, vol. 69, pp. 60-66, 2018.

[15] F. Shirazi, F. Sharif, Z. Molazem, and M. Alborzi, "Dynamics of self-directed learning in M.Sc. nursing students: a qualitative research," Journal of Advances in Medical Education \& Professionalism, vol. 5, no. 1, pp. 33-41, 2017.

[16] E. Shaala, F. Shabaan, and K. EL-said, "Efficacy of self directed learning program to improve technical Institute of nursing students management competencies," Tanta Scientific Nursing Journal, vol. 15, no. 2, pp. 7-24, 2018.

[17] A. Klunklin, N. Viseskul, A. Sripusanapan, and S. Turale, "Readiness for self-directed learning among nursing students in Thailand," Nursing \& Health Sciences, vol. 12, no. 2, pp. 177-181, 2010.

[18] A.-H. El-Gilany and F. E. S. Abusaad, "Self-directed learning readiness and learning styles among Saudi undergraduate nursing students," Nurse Education Today, vol. 33, no. 9, pp. 1040-1044, 2013.

[19] E. Ü. Avdal, "The effect of self-directed learning abilities of student nurses on success in Turkey," Nurse Education Today, vol. 33, no. 8, pp. 838-841, 2013.

[20] B. N. Phillips, B. J. Turnbull, and F. X. He, "Assessing readiness for self-directed learning within a non-traditional nursing cohort," Nurse Education Today, vol. 35, no. 3, pp. e1-e7, 2015.

[21] A. Izadi, M. Barkhordari, Z. Shojai, and M. Zaheri, "Selfdirected learning readiness in baccalaureate nursing students in different academic years and its relationship with anxiety and self-esteem," Journal of Nursing Education, vol. 2, no. 4, pp. 80-89, 2014.

[22] S. Loeng, "Self-directed learning: a core concept in adult education," Education Research International, vol. 2020, Article ID 3816132, 12 pages, 2020.

[23] A. Arsic, Self-Directed Learning in an International Baccalaureate High School Class, Concordia University, Montreal, Canada, 2014.

[24] T. M. Gureckis and D. B. Markant, "Self-directed learning: a cognitive and computational perspective," Perspectives on Psychological Science, vol. 7, no. 5, pp. 464-481, 2012.

[25] J. P. Lantolf, "Introducing sociocultural theory," Sociocultural Theory and Second Language Learning, vol. 1, pp. 1-26, 2000.

[26] N. Rahmani, A. Salehi, H. Molavi Vardanjani, M. Marzban, and A. Behbood, "Using STROBE checklist to assess the reporting quality of observational studies affiliated with Shiraz university of medical sciences, and its correlates: a scientometric study from Iran," Scientometrics, vol. 122, no. 2, pp. 989-1001, 2020.

[27] R.-Z. Luo, X.-H. Zhang, C.-M. Zhang, and Y.-H. Liu, "Impact of self-directed learning readiness and learning attitude on problem-solving ability among Chinese undergraduate nursing students," Frontiers of Nursing, vol. 6, no. 2, pp. 143-150, 2019.

[28] X.-H. Zhang, L.-N. Meng, H.-H. Liu et al., "Role of academic self-efficacy in the relationship between self-directed learning readiness and problem-solving ability among nursing students," Frontiers of Nursing, vol. 5, 2018. 
[29] M. Örs, "The self-directed learning readiness level of the undergraduate students of midwife and nurse in terms of sustainability in nursing and midwifery education," Sustainability, vol. 10, no. 10, p. 3574, 2018.

[30] N. Ertug and S. Faydali, "Investigating the relationship between self-directed learning readiness and time management skills in Turkish undergraduate nursing students," Nursing Education Perspectives, vol. 39, no. 2, pp. E2-E5, 2018.

[31] H. A. Alharbi, "Readiness for self-directed learning: how bridging and traditional nursing students differs?" Nurse Education Today, vol. 61, pp. 231-234, 2018.

[32] H. A. E. Alkorashy and N. E. A. Assi, "Bachelor nursing students readiness for self-directed learning in a Saudi university: a survey-based study," Asian Journal of Nursing Education and Research, vol. 7, no. 1, pp. 66-72, 2017.

[33] B. Arkan, E. Ü Avdal, and H. Y. Sari, "Locus of control and self-directed learning relation on nursing students," International Journal of Caring Sciences, vol. 9, no. 2, p. 514, 2016.

[34] Z. Ghahremani, K. Kamali, and P. Bageri, "Relationship learning styles with self-directed learning readiness among nursing students of nursing and midwifery Zanjan university of medical sciences in 2014," Journal of Medical Education Development, vol. 8, no. 18, pp. 61-72, 2015.

[35] H. B. Yuan, B. A. Williams, J. B. Fang, and D. Pang, "Chinese baccalaureate nursing students' readiness for self-directed learning," Nurse Education Today, vol. 32, no. 4, pp. 427-431, 2012.

[36] L. Naeimi, S. Bigdeli, and K. Soltani Arabshahi, "Level of selfdirected learning readiness in medical students," Education Strategies in Medical Sciences, vol. 5, no. 3, pp. 177-181, 2012.

[37] S. Lee, D. H. Kim, and S.-M. Chae, "Self-directed learning and professional values of nursing students," Nurse Education in Practice, vol. 42, Article ID 102647, 2020.

[38] Y. Song, S. Y. Yun, S.-A. Kim, E.-K. Ahn, and M. S. Jung, "Role of self-directed learning in communication competence and self-efficacy," Journal of Nursing Education, vol. 54, no. 10, pp. 559-564, 2015.

[39] T. L. Levett-Jones, "Self-directed learning: implications and limitations for undergraduate nursing education," Nurse Education Today, vol. 25, no. 5, pp. 363-368, 2005.

[40] M. H. Murad, F. Coto-Yglesias, P. Varkey, L. J. Prokop, and A. L. Murad, "The effectiveness of self-directed learning in health professions education: a systematic review," Medical Education, vol. 44, no. 11, pp. 1057-1068, 2010.

[41] A. Saban and A. Saban, "An investigation of elementary school teaching department students' metacognition awareness and motivation in terms of some socio-demographic variables," Ege Üniversitesi Eğitim Dergisi, vol. 1, no. 9, pp. 35-58, 2008.

[42] Ö. Aydemir, "The learning strategies and success failure attributions of primary school 2nd level students that they use in English lesson," Unpublished Master's Rhesis, Trakya University Institute of Social Sciences, Edirne, Turkey, 2007.

[43] C. E. Slater, A. Cusick, and J. C. Y. Louie, "Explaining variance in self-directed learning readiness of first year students in health professional programs," BMC Medical Education, vol. 17, no. 1, pp. 207-210, 2017.

[44] A. J. Swart, "Self-directed learning-fashionable among all firstyear African engineering students?" Global Journal of Engineering Education, vol. 20, no. 1, 2018.

[45] E. Izci and S. Koç, "The evaluation of the teacher candidates' views on the lifelong learning," Adryaman University Social Sciences Institute Journal, vol. 5, no. 9, pp. 101-114, 2012.
[46] İ. A. Tekkol and M. Demirel, "An investigation of self-directed learning skills of undergraduate students," Frontiers in Psychology, vol. 9, p. 2324, 2018.

[47] C. Y. R. Loh and T. C. Teo, "Understanding Asian students learning styles, cultural influence and learning strategies," Journal of Education \& Social Policy, vol. 7, no. 1, pp. 194-210, 2017.

[48] B. Eilisha, Effectiveness of Working Individually Versus Cooperative Groups: A Classroom-Based Research Project, University of Pennsylvania, Philadelphia, PA, USA, 2007.

[49] C. Zhu, M. Valcke, and T. Schellens, "A cross-cultural study of Chinese and Flemish university students: do they differ in learning conceptions and approaches to learning?" Learning and Individual Differences, vol. 18, no. 1, pp. 120-127, 2008.

[50] J. Heckhausen, Invited Commentary: Societal Constraints and Individual Agency: Navigating Educational Transitions for Upward Mobility, Springer, Berlin, Germany, 2020.

[51] L. Colodro-Conde, F. Rijsdijk, M. J. Tornero-Gómez, J. F. Sánchez-Romera, and J. R. Ordoñana, "Equality in educational policy and the heritability of educational attainment," PLoS One, vol. 10, no. 11, Article ID e0143796, 2015. 